\title{
Oxygen Vacancies at Grain Boundaries in Doubly-Doped Ceria Determined using EELS
}

\author{
W. J. Bowman, J. Zhu, Z. Hussaini, P. A. Crozier
}

School for the Engineering of Matter, Transport, and Energy, Arizona State University, Tempe, Arizona 85287-6106

In oxygen conducting ceramics such as cerium-based oxides, $\mathrm{O}^{2-}$ diffusion occurs via vacancy hopping. The vacancy concentration can be modulated through doping with aliovalent cations such as $\mathrm{Gd}^{3+}$ or $\mathrm{Pr}^{3+}$. Sluggish ionic conductivity in these electrolytes has been attributed to various defects which increase the activation energy for anion migration. The association of mobile oxygen vacancies with dopant cations, and the presence of highly resistive grain boundaries in polycrystalline electrolytes are well-accepted mechanisms which degrade total ionic conductivity [1]. The predominant explanation for high grain boundary resistivity in ceramics of high purity is the space charge double layer (SCDL) which results in vacancy-depleted regions emanating from grain boundary cores [2]. Recently it was shown that addition of $0-2$ at $\%$ transition metal (TM) ions such as $\mathrm{Cr}, \mathrm{Fe}, \mathrm{Ni}$ and $\mathrm{Cu}$ to high purity gadolinium-doped ceria (GDC) enhanced grain boundary electrical conductivity by as much as 15 times by reducing the SCDL potential barrier [3]. Here we use a combination of impedance spectroscopy (EIS) and electron energy-loss spectroscopy (EELS) to characterize the electrical conductivity and vacancy concentration of grain boundaries in ceria doubly-doped with Gd and Pr.

Ceramic electrolytes of compositions $\mathrm{Ce}_{0.85} \mathrm{Gd}_{0.11} \operatorname{Pr}_{0.04} \mathrm{O}_{2-\delta}(G P D C)$ and $\mathrm{Ce}_{0.8} \mathrm{Gd}_{0.2} \mathrm{O}_{2-\delta}$ (GDC) were prepared using a spray drying approach together with traditional ceramic processing techniques. Impedance spectroscopy was performed using a Gamry Reference 3000 potentiostat. Experimental monochromated EELS were acquired using a Nion UltraSTEM100. To facilitate interpretation of the EELS data, FEFF codes [4] were employed to simulate the O K-edge spectra as a function of oxygen vacancy concentration.

Fig. A shows results of a FEFF calculation of the $\mathrm{O}$ K-edge in $\mathrm{CeO}_{2}$ as a function of $\mathrm{O}^{2-}$ vacancy concentration. In this model, the vacancies were randomly distributed throughout the fluorite structure. These simulations indicate a decrease in the first peak in the $\mathrm{O}-\mathrm{K}$ edge fine structure with increasing vacancy concentration. Figs. $\mathrm{B}$ and $\mathrm{C}$ show experimental $\mathrm{Ce} \mathrm{M}_{45}$ and $\mathrm{O}-\mathrm{K}$ edge near edge structure acquired at the edge and center of $\mathrm{CeO}_{2}$ particle. The $\mathrm{Ce}$ white line intensity switch is characteristic of $\mathrm{Ce}^{4+}$ reduction to $\mathrm{Ce}^{3+}$ and in this case is accompanied by a drop in the first $\mathrm{O}$ K-edge peak similar to that in the calculated spectra in (A). Electrical conductivity data in fig. D shows that the grain boundary conductivity is an order of magnitude higher in GPDC compared to GDC. This is significant because it has been shown that GDC with $20 \mathrm{cat}^{\circ} \mathrm{Gd}^{3+}$ exhibits the highest grain boundary conductivity of all of the $\mathrm{Gd}_{\mathrm{x}} \mathrm{Ce}(1-\mathrm{x}) \mathrm{O}_{2-\delta}(\mathrm{x}<0.20)$ family of oxides [3]. Figs. $\mathrm{E}$ and $\mathrm{F}$ show $\mathrm{Ce}$ white lines and $\mathrm{O} \mathrm{K}$-edge fine structure acquired at a grain boundary and grain interior in GPDC. In this case the drop in the first $\mathrm{O}$ $\mathrm{K}$-edge peak is not accompanied by a Ce white line intensity switch.

In this work we will also probe and correlate the cation distribution, the variations in cation oxidation state and the $\mathrm{O} \mathrm{K}$-edge fine structure to elucidate the vacancy environment at grain boundaries in $\mathrm{Gd} / \mathrm{Pr}$ doubly-doped $\mathrm{CeO}_{2}$ electrolytes. These measurements coupled with macroscopic characterization of grain boundary conductivity via impedance spectroscopy will be used to correlate atomic-level grain boundary structure and chemistry with bulk electrical properties. Moreover, we aim to further refine our 
FEFF calculations while also improving the quantitative robustness of our experimental approach to determining the distribution of $\mathrm{O}^{2-}$ vacancies near grain boundaries.

\section{References}

1. Fergus, J., et al., eds. Solid oxide fuel cells: materials properties and performance. CRC press, 2008.

2. Guo, X., et al., Journal of The Electrochemical Society, 148 (3) E121-E126 (2001)

3. Avila-Paredes, H.J., et al., Solid State Ionics 177 (2006) 3075-3080.

4. Rehr, J.J., et al., Physical Chemistry Chemical Physics, 2010.12 (21) 5503-5513

5. We thank the National Science Foundation's Graduate Research Fellowship Program (DGE-

1311230), NSF DMR 1308085, and ASU/NASA Space Grant Program for funding this work. We also gratefully acknowledge support of ASU's John M. Cowley Center for High Resolution Electron Microscopy and Kevin Jorrisen of the University of Washington for help with FEFF codes.
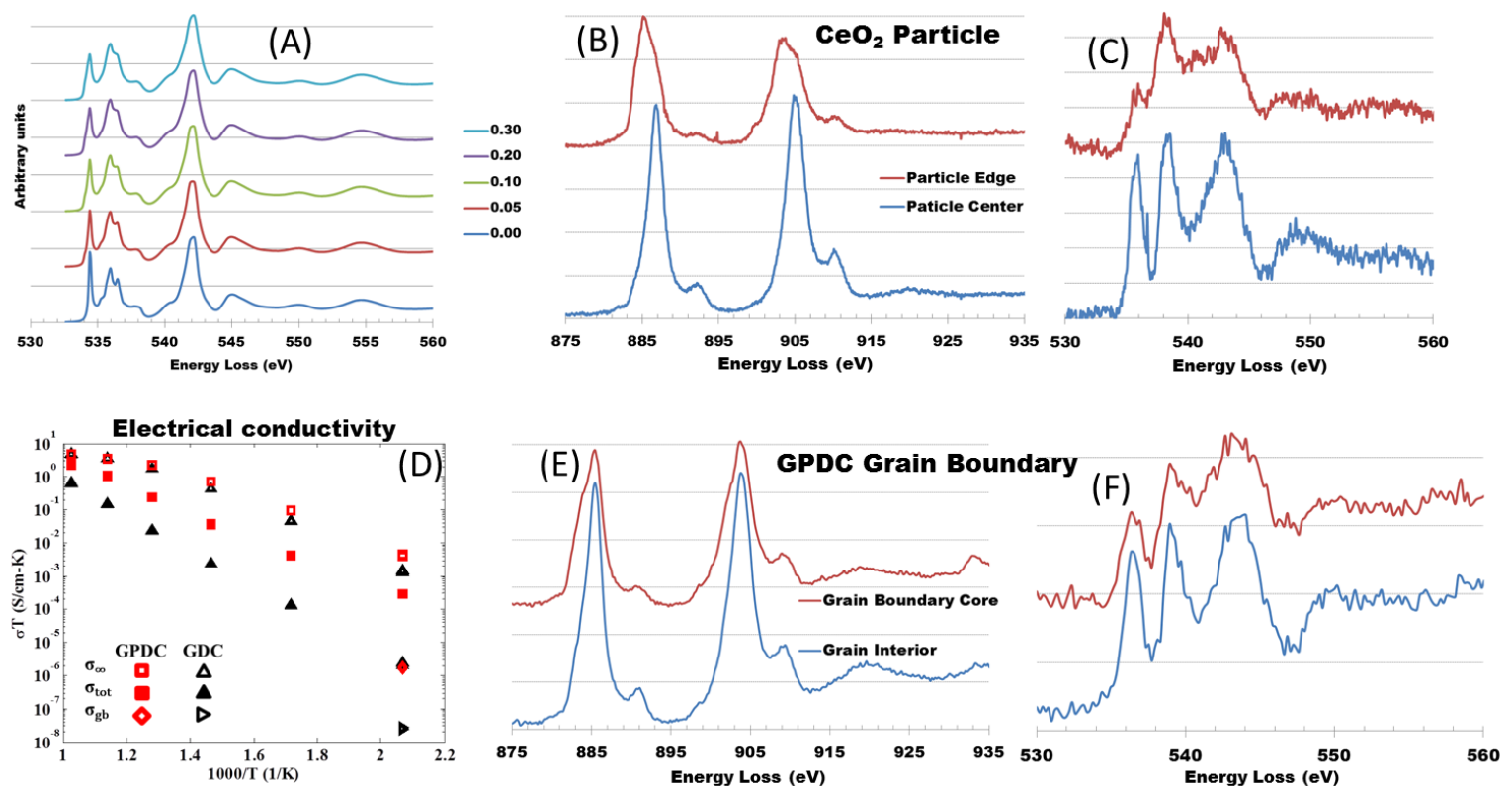

Fig. A: Calculation of EELS O K-edge near-edge structure as a function of $\mathrm{O}^{2-}$ vacancy in $\mathrm{CeO}_{2}$. The intensity of the first peak drops with increasing vacancy concentration.

Figs. B \& C: Experimental $\mathrm{Ce}_{45}$ white lines and $\mathrm{O}$ K-edge near edge structure acquired at the edge and center of a $\mathrm{CeO}_{2}$ particle.

Fig. D: Conductivity data showing the much higher GPDC grain boundary conductivity, $\sigma_{\mathrm{gb}}(\diamond)$. Figs. E \& F: Experimental $\mathrm{Ce}_{45}$ white lines and $\mathrm{O} \mathrm{K}$-edge near edge structure acquired at a grain boundary and grain interior in GPDC. 